\title{
Patients with Gastric Antral Vascular Ectasia (GAVE) Are at a Higher Risk of Gastrointestinal Bleeding in the Absence of Cirrhosis
}

\author{
Jennifer Wang ${ }^{1}$, Jonathan G. Stine* ${ }^{2}$, Scott L. Cornella ${ }^{1}$, Curtis K. Argo ${ }^{2}$ and Steven M. Cohn ${ }^{2}$ \\ ${ }^{1}$ Department of Medicine, University of Virginia, Charlottesville, Virginia, USA; ${ }^{2}$ Division of Gastroenterology and Hepatology, \\ University of Virginia, Charlottesville, Virginia, USA
}

\begin{abstract}
Background and Aims: Gastric antral vascular ectasia (GAVE) is commonly found in patients with cirrhosis, but it is also associated with other diseases in the absence of cirrhosis. Whether GAVE confers a different severity of gastrointestinal (GI) bleeding between patients with and without cirrhosis remains unknown. We aim to examine whether there is a difference in clinically significant GI bleeding due to GAVE in patients with or without cirrhosis. Methods: This is a retrospective case-control study of patients who were diagnosed with GAVE between January 2000 and June 2014. Patients were categorized into cirrhosis and noncirrhosis groups, and those with an additional GI bleeding source were excluded. Univariate comparisons and multivariable models were constructed using logistic regression. Results: In total, 110 patients diagnosed with GAVE on esophagogastroduodenoscopy (EGD) were included in our analysis; 84 patients had cirrhosis (76.4\%) and $26(23.6 \%)$ did not. Active GI bleeding was more prevalent in patients without cirrhosis $(63.4 \%$ vs. $32.1 \%, p=0.003$ ) despite similar indications for EGD, and endoscopic treatment with argon plasma coagulation (APC) was required more often in this group, approaching statistical significance $(27 \%$ vs. $10.7 \%, p=0.056)$. There was no difference in bleeding severity, as evidenced by similar re-bleeding rates, surgery, or death attributed to uncontrolled bleeding. The strongest independent risk factor for GI bleeding was the absence of cirrhosis (odds ratio (OR): 5.151 (95\% confidence interval (CI): 1.08-24.48, $p=0.039$ ). Conclusions: Patients with GAVE in the absence of cirrhosis are at higher risk for active GI bleeding and require more frequent endoscopic treatment than similar patients with
\end{abstract}

Keywords: Gastric antral vascular ectasia; Liver disease; Portal hypertension Endoscopy.

Abbreviations: $\mathrm{AIH}$, autoimmune hepatitis; APC, argon plasma coagulation BICAP, bipolar circumactive probe; CI, confidence interval; CKD, chronic kidney disease; DILI, drug induced liver injury; EGD, esophagogastroduodenoscopy ESRD, end-stage renal disease; GAVE, gastric antral vascular ectasia; GI, gastrointestinal; HCV, hepatitis C virus; ICD, International Classification of Diseases Ninth Revision; MELD, Model for End-Stage Liver Disease; NASH, nonalcoholic steatohepatitis; NSAID, nonsteroidal anti-inflammatory drug; OR, odds ratio PPI, proton pump inhibitor; PSC, primary sclerosis cholangitis; VIP, vasoactive intestinal peptide.

Received: 24 August 2015; Revised: 09 October 2015; Accepted: 11 October 2015 DOI: $10.14218 / \mathrm{JCTH} .2015 .00031$.

* Correspondence to: Jonathan G Stine, Division of Gastroenterology and Hepatology, JPA and Lee Street, MSB2145, PO Box 800708, University of Virginia, Charlottesville, VA 22908-0708, USA. Tel: +1-434-243-7741, Fax: +1-434-2447529, E-mail: jgs9f@virginia.edu cirrhosis. It may be worthwhile to treat GAVE in this population even in the absence of active bleeding.

(C) 2015 The Second Affiliated Hospital of Chongqing Medical University. Published by XIA \& HE Publishing Ltd. All rights reserved.

\section{Introduction}

Gastric antral vascular ectasia (GAVE) is an uncommon cause of occult gastrointestinal (GI) bleeding and iron-deficiency anemia, accounting for $4 \%$ of nonvariceal upper GI hemorrhage. ${ }^{1}$ The clinical presentation of GAVE varies from a more indolent, chronic occult GI blood loss requiring serial transfusions to acute GI hemorrhage. ${ }^{1}$ First described by Jabbari et al. in $1984,{ }^{2}$ GAVE is also named watermelon stomach due to the gross endoscopic appearance of red stripes radiating from the antrum converging on the pylorus. GAVE is commonly associated with cirrhosis of the liver and, in particular, portal hypertension; ${ }^{3}$ however, it has also been associated with such underlying chronic diseases as autoimmune connective tissue disorders, chronic renal failure, systemic lupus erythematosus, and diabetes mellitus (Table 1 ). ${ }^{4-7}$ In patients with cirrhosis, GAVE is found more commonly in patients with the most advanced liver disease. ${ }^{8}$ GAVE has two distinct endoscopic forms, diffused or punctate, which is more commonly associated with cirrhosis ${ }^{9-10}$ and striped (linear), which typically occurs in the absence of cirrhosis. ${ }^{10}$ Despite the difference in endoscopic appearance, the histopathologic parameters of GAVE in patients with cirrhosis are identical to patients without cirrhosis ${ }^{11}$ and is characterized by a combination of four findings: vascular ectasia of mucosal capillaries, focal thrombosis, spindle cell proliferation, and fibrohyalinosis. ${ }^{12}$ The diagnosis of GAVE can be difficult, as it can be confused with portal hypertensive gastropathy. However, the pathophysiology of these two diseases is entirely different, as GAVE is more likely to be driven by the neurohumoral factors prostaglandin $E$ and possibly gastrin, vasoactive intestinal peptide (VIP), and/or 5-hydroxytryptamine (5-HT), ${ }^{13-16}$ leading to vasodilation and impaired motility. ${ }^{17}$

In general, there is a lack of epidemiologic evidence supporting differences in clinical outcomes in GAVE patients with and without cirrhosis. Moreover, whether GAVE confers a different severity of GI bleeding remains unknown. One series of 30 patients by Ito et al. observed lower hemoglobin levels at baseline and a greater need for transfusion when comparing 
Table 1. Diseases associated with GAVE in the absence of cirrhosis ${ }^{4,6-9}$

Diabetes mellitus
Chronic renal failure
Coronary artery disease
Sjogren's syndrome
Rheumatoid arthritis
Systemic sclerosis
Calcinosis, Raynaud's, esophageal dysmotility,
scleroderma, telangiectasia (CREST)
Systemic lupus erythematosus
Polymyalgia rheumatic
Bone marrow transplant patient
Acute myelogenous leukemia
Monoclonal gammopathy of undetermined significance
Parkinson disease

patients with GAVE who did not have cirrhosis to those with cirrhosis. ${ }^{6}$ Here, we examined whether there is a difference in clinically significant GI bleeding secondary to GAVE in a cohort of patients with and without cirrhosis.

\section{Methods}

We conducted a retrospective case-control study of patients who were found to have GAVE at the University of Virginia between 1 January 2000 and 30 June 2014 using International Classification of Diseases, Ninth Revision, Clinical Modification (ICD-9) codes. Patients were queried from the Clinical Data Repository, and a primary cohort of patients with GAVE was constructed. Endoscopy reports were reviewed independently by two gastroenterologists/hepatologists. If a disagreement on the diagnosis of GAVE occurred, a third independent gastroenterologist/hepatologist reviewed the report for final decision on inclusion. Endoscopy patients were categorized into cases (cirrhosis) or controls (noncirrhosis). The diagnosis of cirrhosis was based initially by screening ICD-9 codes and confirmed by available histologic sampling by liver biopsy or by imaging studies (both crosssectional and ultrasound). Only patients at or above age 18 were included in the analysis. Patients who had an additional GI bleeding source (e.g. gastroesophageal varices, gastric ulcer, etc.) were excluded. Baseline covariate characteristics were reviewed, including age, gender, race, etiology of liver disease, severity of liver disease based on Model for EndStage Liver Disease (MELD), and Child-Pugh scores, laboratory values [platelet counts, creatinine, blood urea nitrogen, sodium, albumin, International Normalized Ratio (INR), and total bilirubin], GAVE risk factors [hypothyroidism, bone marrow transplant, chronic kidney disease (CKD), end-stage renal disease (ESRD), diabetes, proton pump inhibitor (PPI) use, connective tissue disease], and GI bleeding risk factors [active smoking, active alcohol use, anticoagulant or antiplatelet agent use, and non-steroidal anti-inflammatory drug (NSAID) use]. Primary outcomes were objective evidence of active GI bleeding, which was defined as symptoms of melena, hematochezia, hematemesis, change in hemodynamics, or gross bleeding on esophagogastroduodenoscopy (EGD). Secondary outcomes included number of transfu- sions, baseline hemoglobin, need for endoscopic intervention [including argon plasma coagulation (APC)], rebleeding rates, need for surgery, and death.

\section{Statistical analysis}

Patients with GAVE and cirrhosis were compared statistically to those without cirrhosis in multiple factors, including demographics, laboratory values, GAVE risk factors, and GI bleeding risk factors. Univariate comparisons were performed using the Student-t test, Wilcoxon sign rank test, chi-square test, and Fisher exact test, as appropriate. When the normality of continuous variables was not assumed or when the equality of variances was not observed, logarithmic transformation was done. Multivariable models were constructed to assess statistical associations and risk factors for the development of bleeding from GAVE using logistic regression and analysis of the maximum likelihood estimates. Odds ratios (OR) were calculated and were used as an estimation of risk under the rare disease assumption. Individual factors were included in the multivariable model if they were statistically significant $(p<0.20)$ in the univariate analysis, were clinically important, or have been shown in the literature to be important. ${ }^{18,19}$ Multiple iterations of the model were performed. Variables included in the final iteration of the model included presence or absence of cirrhosis, platelet count, hypothyroidism, diabetes, PPI use, NSAID use, hemoglobin, type of GAVE, EGD indication, and CKD. All data set manipulation and statistical analyses were carried out using SAS (version 9.4, Cary, USA). No data involving prisoners were included in this analysis. All statistical tests for significance were two sided and a significance level of $p \leq 0.05$ was considered statistically significant. Institutional review board approval was obtained.

\section{Results}

A total of 478 patients underwent EGD and were diagnosed with GAVE between January 2000 and June 2014. Of those, 110 met the inclusion criteria for our analysis. Eighty-four patients were in the cirrhosis group $(76.4 \%)$ and 26 were in the noncirrhosis group (23.6\%). In the cirrhosis group, alcohol was the most common etiology of cirrhosis (34.5\%), and the mean MELD score was 13.3. In the noncirrhosis group, the most common concomitant disorder was diabetes mellitus $(n=12)$, followed by CKD $(n=8)$. The less commonly associated conditions were bone marrow transplantation recipients, hypothyroidism, and connective tissue disease. Baseline characteristics amongst patients with and without cirrhosis were in general similar with several exceptions (Table 2). Of the covariates analyzed, the platelet count and albumin level in patients without cirrhosis were higher than patients with cirrhosis $(p<0.05)$. Those without cirrhosis also had an increased prevalence of CKD (Table 2). The indication for initial EGD was also similar between the two groups when dichotomized to those performed for suspected GI blood loss versus other indications. Use of anticoagulant or antiplatelet agents was rare amongst our patients, and only one patient in the noncirrhosis group was on an anticoagulant.

In univariate analysis, active GI bleeding was more common in the noncirrhosis group than the cirrhosis group (63.4\% vs. $32.1 \%, p=0.003$ ) (Table 3 ). There was no statistically significant difference in the baseline levels of hemoglobin or hematocrit between the two groups. The number of 
Wang J. et al: GI bleeding in GAVE patients without cirrhosis

Table 2. Baseline characteristics of patients with GAVE at the time of endoscopic diagnosis

\begin{tabular}{|c|c|c|c|}
\hline & $\begin{array}{l}\text { Cirrhosis } \\
(n=84,76.4 \%)\end{array}$ & $\begin{array}{l}\text { No cirrhosis } \\
(n=26,23.6 \%)\end{array}$ & $p$ value \\
\hline \multicolumn{4}{|c|}{ Recipient characteristics } \\
\hline Age $(95 \% \mathrm{CI})$ & $59.7(57.0-62.3)$ & $63.3(59.3-67.3)$ & NS \\
\hline Female gender & $40(47.6 \%)$ & $14(53.8 \%)$ & NS \\
\hline Male gender & $44(52.4 \%)$ & $12(46.2 \%)$ & NS \\
\hline \multicolumn{4}{|l|}{ Race } \\
\hline Caucasian & $70(83.3 \%)$ & $22(84.6 \%)$ & NS \\
\hline African American & $5(6.7 \%)$ & $2(7.7 \%)$ & NS \\
\hline Other & $3(3.9 \%)$ & $0(0.0 \%)$ & NS \\
\hline Unknown & $6(7.1 \%)$ & $2(7.7 \%)$ & NS \\
\hline \multicolumn{4}{|l|}{ Cirrhosis etiology } \\
\hline Alcohol & $29(34.5 \%)$ & \multirow{8}{*}{ N/A } & \multirow{8}{*}{ N/A } \\
\hline $\mathrm{NASH}$ & $24(28.6 \%)$ & & \\
\hline $\mathrm{HCV}$ & $21(25.0 \%)$ & & \\
\hline Cryptogenic & $8(9.5 \%)$ & & \\
\hline PBC & $3(3.6 \%)$ & & \\
\hline PSC & $3(3.6 \%)$ & & \\
\hline $\mathrm{AIH}$ & $2(2.4 \%)$ & & \\
\hline Chronic DILI & $1(1.2 \%)$ & & \\
\hline \multicolumn{4}{|c|}{ Severity of liver disease } \\
\hline $\begin{array}{l}\text { Model for End State Liver Disease } \\
\text { (MELD) score }(95 \% \mathrm{CI})\end{array}$ & $13.3(11.8-14.8)$ & N/A & $\mathrm{N} / \mathrm{A}$ \\
\hline \multicolumn{2}{|l|}{ Child Pugh Score } & \multirow{4}{*}{$\mathrm{N} / \mathrm{A}$} & \multirow{4}{*}{ N/A } \\
\hline Class A & $36(42.9 \%)$ & & \\
\hline Class B & $37(44.0 \%)$ & & \\
\hline Class C & $11(13.1 \%)$ & & \\
\hline \multicolumn{4}{|c|}{ Indications for initial EGD } \\
\hline Suspected GI blood loss & $27(32.1 \%)$ & $18(69.2 \%)$ & 0.001 \\
\hline \multicolumn{4}{|c|}{ Laboratory values } \\
\hline Platelet count, $10^{9} / \mathrm{L}$, mean $(95 \% \mathrm{CI})$ & $120.9(100.3-141.4)$ & $226.3(179.2-273.3)$ & $<0.001$ \\
\hline Creatinine, mg/dL, mean ( $95 \% \mathrm{CI})$ & $1.26(1.04-1.47)$ & $1.85(1.08-2.62)$ & NS \\
\hline BUN, mg/dL, mean $(95 \% \mathrm{CI})$ & $19.6(16.5-22.7)$ & $30.0(19.6-40.5)$ & NS \\
\hline Sodium, meq/L, mean ( $95 \% \mathrm{CI})$ & $137.2(136.4-138.0)$ & $138.8(137.2-140.5)$ & NS \\
\hline Albumin, $\mathrm{g} / \mathrm{dL}$, mean $(95 \% \mathrm{CI})$ & $3.38(3.22-3.55)$ & $3.85(3.59-4.12)$ & 0.001 \\
\hline INR, mean $(95 \% \mathrm{CI})$ & $1.36(1.29-1.42)$ & $1.43(1.12-1.73)$ & NS \\
\hline $\begin{array}{l}\text { Total bilirubin, } \mathrm{mg} / \mathrm{dL} \text {, mean } \\
(95 \% \mathrm{CI})\end{array}$ & $2.60(1.46-3.75)$ & $1.27(0.07-2.48)$ & NS \\
\hline \multicolumn{4}{|c|}{ GAVE risk factors } \\
\hline Hypothyroid & $6(7.1 \%)$ & $1(3.9 \%)$ & NS \\
\hline BM transplant & $0(0.0 \%)$ & $1(3.9 \%)$ & NS \\
\hline $\begin{array}{l}\text { Chronic Kidney Disease (including } \\
\text { End-Stage Renal Disease) }\end{array}$ & $5(6.0 \%)$ & $8(30.8 \%)$ & 0.002 \\
\hline Diabetes & $43(51.2 \%)$ & $12(46.2 \%)$ & NS \\
\hline PPI use & $51(62.2 \%)$ & $18(69.2 \%)$ & NS \\
\hline Connective tissue disease & $1(1.2 \%)$ & $2(7.7 \%)$ & NS \\
\hline
\end{tabular}


Wang J. et al: GI bleeding in GAVE patients without cirrhosis

Table 2. (continued)

\begin{tabular}{llll}
\hline & $\begin{array}{l}\text { Cirrhosis } \\
(\mathrm{n}=84,76.4 \%)\end{array}$ & $\begin{array}{l}\text { No cirrhosis } \\
(\mathrm{n}=26,23.6 \%)\end{array}$ & $p$ value \\
\hline \multicolumn{1}{l}{$\begin{array}{l}\text { GI bleeding risk factors } \\
\text { Active alcohol use }\end{array}$} & $24(29.3 \%)$ & $8(32.0 \%)$ & NS \\
$\begin{array}{l}\text { Anticoagulant or antiplatelet agent } \\
\text { use }\end{array}$ & $27(32.1 \%)$ & $8(30.8 \%)$ & NS \\
NSAID use & $0(0 \%)$ & $2(7.7 \%)$ & 0.010 \\
\hline
\end{tabular}

AIH, autoimmune hepatitis; DILI, drug induced liver injury; HCV, hepatitis C virus; NASH, nonalcoholic steatohepatitis; NS, not significant; NSAID, nonsteroidal antiinflammatory inhibitor; PBC, primary biliary sclerosis; PPI, proton pump inhibitor; PSC, primary sclerosis cholangitis.

blood transfusions required was also similar. The endoscopic findings of the patient groups are shown in Table 4. The proportion of patients with cirrhosis who had the striped (linear) form of vascular ectasia was $68.0 \%$ compared to $46.2 \%$ in the noncirrhosis group $(p=0.048)$. Endoscopic treatment with APC was required more often in patients without cirrhosis than patients with cirrhosis and approached statistical significance $(27.0 \%$ vs. $10.7 \%, p=0.056)$. However, there was no difference between the two groups in bleeding severity, as evidenced by similar rates of rebleeding, need to escalate therapy to surgery, and death. Death from any cause was relatively rare overall $(n=2)$ in this population. No patient had a death attributable to GI hemorrhage.

After adjusting for the presence confounders, our multivariable regression analysis (Table 5 ) showed that absence of cirrhosis was the strongest predictor of active bleeding from GAVE with OR: 5.151 ( $95 \%$ CI: $1.084-24.480, p=0.039$ ). No other variable included in our model, except for INR, was significantly associated with an increased risk of active GI bleeding from GAVE. The model was deemed significant ( $p=0.041$ for likelihood ratio test statistic) and had good predictability, as the c-statistic of the model was 0.85 .

\section{Discussion}

GAVE is an uncommon cause of acute upper GI bleeding and is traditionally thought to be associated largely with cirrhosis. In our retrospective case-control study, we have found the opposite. Patients with GAVE in the absence of cirrhosis had a higher risk of developing active GI bleeding and required endoscopic treatment more frequently than those with cirrhosis. In fact, after adjusting for the presence of comorbidities associated with GAVE, risk factors for GI bleeding and coagulopathy, the only significant predictor for clinically evident GI bleeding in the setting of GAVE was the absence of cirrhosis. However, there was no difference in the severity of GI bleeding measured by levels of hemoglobin and hematocrit or the total number of transfusions prior to presentation. Electrocoagulation was the mainstay therapy used in most cases, and the outcome of the therapy was similar between the two groups. Irrespective of cirrhosis, patients had a similar response to treatment in terms of rebleeding events, escalation of treatment to surgery, and mortality. Death from GAVE was rare. Only two patients in our study died from causes not related to GAVE. No patients died of blood loss in the setting of GAVE.

Our findings confirmed those of Ito et $a l^{6}$ and expanded them on a much larger scale. In a retrospective study of 30 patients, of which 25 had cirrhosis, they observed that patients with GAVE in the absence of cirrhosis had lower hemoglobin levels. However, they failed to control for the presence of confounders in their analysis, and the indication for endoscopy in their patient population included variceal screening, which could introduce lead-time bias in the cirrhosis group in which endoscopies were routinely performed to screen for esophageal varices and could recognize GAVE in asymptomatic patients. In our study, we reviewed each patient's indication for initial EGD, which included esophageal varices screening, chronic anemia, and overt GI bleeding. After excluding esophageal varices screening in asymptomatic patients and only

Table 3. Bleeding severity, univariate analysis

\begin{tabular}{|c|c|c|c|}
\hline & $\begin{array}{l}\text { Cirrhosis } \\
(n=84,76.4 \%)\end{array}$ & $\begin{array}{l}\text { No cirrhosis } \\
(n=26,23.6 \%)\end{array}$ & $p$-value \\
\hline \multicolumn{4}{|c|}{ Bleeding severity } \\
\hline Overt bleeding & $27(32.1 \%)$ & $17(63.4 \%)$ & 0.003 \\
\hline Heart rate & $75.0(69.7-80.3)$ & $81.8(70.5-93.0)$ & NS \\
\hline Systolic blood pressure & $125.7(118.8-132.7)$ & $134.1(121.4-146.8)$ & NS \\
\hline Transfusion (units) & $1.08(0.33-1.83)$ & $1.37(0.66-2.14)$ & NS \\
\hline Hemoglobin & $11.5(11.0-12.0)$ & $10.7(9.5-11.8)$ & NS \\
\hline Hematocrit & $34.4(32.9-35.8)$ & $32.6(29.6-35.7)$ & NS \\
\hline
\end{tabular}

NS, not significant. 
Wang J. et al: GI bleeding in GAVE patients without cirrhosis

Table 4. Outcomes, univariate analysis

\begin{tabular}{|c|c|c|c|}
\hline & $\begin{array}{l}\text { Cirrhosis } \\
(n=84,76.4 \%)\end{array}$ & $\begin{array}{l}\text { No Cirrhosis } \\
(n=26,23.6 \%)\end{array}$ & $p$-value \\
\hline \multicolumn{4}{|c|}{ Endoscopic features and treatment } \\
\hline Linear GAVE & $51(68.0 \%)$ & $12(46.2 \%)$ & 0.048 \\
\hline Punctate GAVE & $25(33.3 \%)$ & $14(53.8 \%)$ & NS \\
\hline APC & $9(10.7 \%)$ & $7(27.0 \%)$ & NS \\
\hline BICAP & $3(3.57 \%)$ & $3(11.5 \%)$ & NS \\
\hline Epinephrine/BICAP & $0(0.0 \%)$ & $1(3.9 \%)$ & NS \\
\hline \multicolumn{4}{|c|}{ Outcomes } \\
\hline Surgery & $0(0 \%)$ & $0(0 \%)$ & NS \\
\hline $\begin{array}{l}\text { Death attributable to uncontrolled } \\
\text { bleeding }\end{array}$ & $1(1.2 \%)$ & $1(3.9 \%)$ & NS \\
\hline Rebleed $\leq 72$ hours & $1(1.2 \%)$ & $1(3.9 \%)$ & NS \\
\hline Rebleed $>72$ hours & $10(12.9 \%)$ & $6(23.1 \%)$ & NS \\
\hline
\end{tabular}

APC, argon plasma coagulation; BICAP, bipolar circumactive probe; GAVE, gastric antral vascular ectasia; NS, not significant.

comparing symptomatic patients, we found that EGD was done more frequently in the cirrhosis group in the setting of anemia or overt GI bleeding than that of the noncirrhosis group. The small sample size of symptomatic patients is certainly a limitation; however, it reflects the fact that GAVE associated nonvariceal GI bleeding is a rare event. Regardless, Ito et al. ${ }^{6}$ argued that there was an inherent difference in either the pattern of GAVE (punctate vs. linear) or the presence or absence of cirrhosis, but they could not distinguish the independent contribution of these factors with their analysis. Our multivariable analysis argues against this, as the pattern of GAVE did not reach statistical significance in predicting a patient's likelihood of having active GI bleeding. In addition, the two patterns of GAVE were indistinguishable histologically, suggesting that the two entities may be more similar than was previously realized. Yet, there is a difference in circulating neurohumoral factors (prostaglandin E, VIP, and 5-HT) between patients with cirrhosis and those without. ${ }^{13-16}$ Further study is needed to define the clinical importance of these biomarkers and to investigate any clinical utility.
Our study had several limitations. While it contained a larger sample size than previous studies and was well powered, it was nonetheless retrospective. Missing data was also problematic as roughly $3 / 4$ of patients who underwent EGD and were coded as having GAVE were excluded for this reason. This could have introduced bias either towards or away from the null hypothesis. Another limitation of our study was a lack of follow-up, as our rebleeding rates were calculated at $72 \mathrm{~h}$ due to limitations in our retrospective dataset. This is problematic with GAVE, in particular, as these patients often required multiple treatment sessions (30-60\%), sometimes months after their first treatment. ${ }^{20-22}$

\section{Conclusions}

Based on our findings and the work of others, we argue that the dogma of GAVE as largely associated with cirrhosis and/or portal hypertension may in fact be incorrect and that GAVE should be considered on the differential diagnosis for any patient with objective evidence of acute upper GI

Table 5. Multivariable analysis using logistic regression and maximum likelihood estimates

\begin{tabular}{llll}
\hline Variable & Odds ratio & $95 \%$ confidence interval & $p$ value \\
\hline No cirrhosis & 5.15 & $1.08-24.48$ & 0.039 \\
Log platelet count & 1.34 & $0.53-3.35$ & NS \\
INR & 8.75 & $1.33-57.42$ & 0.024 \\
Active alcohol use & 0.86 & $0.28-2.60$ & NS \\
Hypothyroid & 0.79 & $0.13-4.92$ & NS \\
Diabetes & 0.93 & $0.35-2.54$ & NS \\
PPI use & 0.68 & $0.24-1.96$ & NS \\
NSAID use & 1.05 & $0.24-4.52$ & NS \\
Chronic kidney disease & 3.49 & $0.63-19.23$ & NS \\
Linear GAVE & 1.38 & $0.43-4.36$ & NS \\
\hline
\end{tabular}

GAVE, gastric antral vascular ectasia; INR, international normalized ratio; NS, not significant; NSAID, non-steroidal anti-inflammatory drug; PPI, proton pump inhibitor. 
bleeding. If GAVE is diagnosed endoscopically, a thorough investigation for not only cirrhosis of the liver but also other noncirrhosis disease processes associated with GAVE should ensue. Future studies should focus on the mechanistic differences between GAVE patients with and without cirrhosis in order to determine if neurohumoral factors differ as a potential explanation for the differences observed clinically.

\section{Acknowledgments}

This study was funded by the National Institute of Diabetes and Digestive and Kidney Diseases of the National Institutes of Health under award number T32DK007769. We would like to acknowledge Brian McNichols and Joseph Hall for their contributions to data acquisition. We would like to acknowledge Neeral Shah for his manuscript review.

\section{Conflict of interest}

None.

\section{Author contributions}

Writing the paper (JW, JGS, SLC, CKA, SMC).

\section{References}

[1] Dulai GS, Jensen DM, Kovacs TO, Gralnek IM, Jutabha R. Endoscopic treatment outcomes in watermelon stomach patients with and without portal hypertension. Endoscopy 2004;36:68-72.

[2] Jabbari M, Cherry R, Lough JO, Daly DS, Kinnear DG, Goresky CA. Gastric antral vascular ectasia: the watermelon stomach. Gastroenterology 1984 87:1165-1170.

[3] Egger C, Kreczy A, Kirchmair R, Waldenberger P, Jaschke W, Vogel W. Gastric antral vascular ectasia with portal hypertension: treatment with TIPSS. Am Gastroenterol 1997;92:2292-2294.

[4] Tobin RW, Hackman RC, Kimmey MB, Durtschi MB, Hayashi A, Malik R, et al. Bleeding from gastric antral vascular ectasia in marrow transplant patients. Gastrointest Endosc 1996;44:223-229.

[5] Gostout C], Viggiano TR, Ahlquist DA, Wang KK, Larson MV, Balm R. The clinical and endoscopic spectrum of the watermelon stomach. J Clin Gastroenterol 1992;15:256-263.
[6] Ripoll C, Garcia-Tsao G. The management of portal hypertensive gastropathy and gastric antral vascular ectasia. Dig Liver Dis 2011;43:345-351.

[7] Ito M, Uchida Y, Kamano S, Kawabata H, Nishioka M. Clinical comparisons between two subsets of gastric antral vascular ectasia. Gastrointest Endosc 2001;53:764-770. doi: 10.1067/mge.2001.113922.

[8] Elkayam O, Oumanski M, Yaron M, Caspi D. Watermelon stomach following and preceding systemic sclerosis. Semin Arthritis Rheum 2000;30:127-131. doi: 10.1053/sarh.2000.9623.

[9] Spahr L, Villeneuve JP, Dufresne MP, Tassé D, Bui B, Willems B, et al. Gastric antral vascular ectasia in cirrhotic patients: absence of relation with portal hypertension. Gut 1999;44:739-742.

[10] Lee FI, Costello F, Flanagan N, Vasudev KS. Diffuse antral vascular ectasia. Gastrointest Endosc 1984;30:87-90.

11] Payen JL, Cales P, Voigt JJ, Barbe S, Pilette C, Dubuisson L, et al. Severe portal hypertensive gastropathy and antral vascular ectasia are distinct entities in patients with cirrhosis. Gastroenterology 1995;108:138-144.

[12] Gilliam JH 3rd, Geisinger KR, Wu WC, Weidner N, Richter JE. Endoscopic biopsy is diagnostic in gastric antral vascular ectasia. The "watermelon stomach". Dig Dis Sci 1989;34:885-888.

[13] Qureshi K, Al-Osaimi AM. Approach to the management of portal hypertensive gastropathy and gastric antral vascular ectasia. Gastroenterol Clin North Am 2014;43:835-847. doi: 10.1016/j.gtc.2014.08.012.

[14] Quintero E, Pique JM, Bombi JA, Bordas JM, Sentis J, Elena M, et al. Gastric mucosal vascular ectasias causing bleeding in cirrhosis. A distinct entity associated with hypergastrinemia and low serum levels of pepsinogen I. Gastroenterology 1987;93:1054-1061.

[15] Saperas E, Perez Ayuso RM, Poca E, Bordas JM, Gaya J. Increased gastric PGE2 biosynthesis in cirrhotic patients with gastric vascular ectasia. Am J Gastroenterol 1990;85:138-144.

[16] Lowes JR, Rode J. Neuroendocrine cell proliferations in gastric antral vascular ectasia. Gastroenterology 1989;97:207-212.

[17] Charneau J, Petit R, Cales P, Dauver A, Boyer J. Antral motility in patients with cirrhosis with or without gastric antral vascular ectasia. Gut 1995;37: 488-492.

[18] Sun GW, Shook TL, Kay GL. Inappropriate use of bivariable analysis to screen risk factors for use in multivariable analysis. J Clin Epidemiol 1996;49:907916. doi: 10.1016/0895-4356(96)00025-X.

[19] Bursac Z, Gauss CH, Williams DK, Hosmer DW. Purposeful selection of variables in logistic regression. Source Code Biol Med 2008;3:17. doi: 10. 1186/1751-0473-3-17.

[20] Yusoff I, Brennan F, Ormonde D, Laurence B. Argon plasma coagulation for treatment of watermelon stomach. Endoscopy 2002;34:407-410.

[21] Roman S, Saurin JC, Dumortier J, Perreira A, Bernard G, Ponchon T. Tolerance and efficacy of argon plasma coagulation for controlling bleeding in patients with typical and atypical manifestations of watermelon stomach. Endoscopy 2003;35:1024-1028.

[22] Ghrenassia E, Avouac J, Khanna D, Derk CT, Distler O, Suliman YA, et al. Prevalence, correlates and outcomes of gastric antral vascular ectasia in systemic sclerosis: a EUSTAR case-control study. J Rheumatol 2014;41: 99-105. doi: 10.3899/jrheum.130386. 